\title{
WHOLE BLOOD IONIZED MAGNESIUM: AGE-RELATED DIFFERENCES IN NORMAL VALUES AND CLINICAL IMPLICATIONS OF IONIZED HYPOMAGNESEMIA IN PATIENTS UNDERGOING SURGERY FOR CONGENITAL CARDIAC DISEASE
}

\author{
Ricardo Munoz, MD ${ }^{\mathrm{a}, \mathrm{c}}$ \\ Peter C. Laussen, MBBS ${ }^{\mathrm{a}, \mathrm{b}, \mathrm{c}, \mathrm{d}}$ \\ Guillermo Palacio, MD ${ }^{\mathrm{a}, \mathrm{c}}$ \\ Lynne Zienko, BS ${ }^{\mathrm{b}}$ \\ Gary Piercey, $\mathrm{BS}^{\mathrm{a}}$ \\ David L. Wessel, MD ${ }^{\mathrm{a}, \mathrm{b}, \mathrm{c}, \mathrm{d}}$
}

Objectives: We sought to (1) determine reference values for whole blood ionized magnesium concentrations in newborns, children, and young adults and (2) evaluate the frequency and clinical implications of ionized hypomagnesemia in patients undergoing surgery for congenital heart disease.

Method: We prospectively measured ionized magnesium concentrations in 299 subjects (113 control subjects and 186 patients undergoing surgery for congenital heart disease). Subjects were categorized by age. In the surgical group blood samples were obtained before bypass, during bypass (cooling and rewarming), after bypass, and during admission to the intensive care unit. Ionized hypomagnesemia was defined as ionized magnesium level 2 standard deviations below the mean of control subjects in the same age group. Patients were analyzed, controlling for cardiopulmonary bypass time.

Results: In the control group ionized magnesium concentrations differed by age. Neonates and adults showed lower ionized magnesium concentrations compared with those of other age groups. Infants exhibited the highest ionized magnesium concentration. In the surgical group patients older than 1 month showed a higher proportion of ionized hypomagnesemia compared with that found in neonates at baseline $(P<.001)$, after bypass $(P=.03)$, and at admission to the intensive care unit $(P=.02)$. Controlling for cardiopulmonary bypass time, patients older than 1 month who were hypomagnesemic during bypass showed longer intubation time $(P=.001)$ and longer intensive care stay $(P=.01)$ and tended to have a higher pediatric severity of illness score on intensive care admission $(P=.14)$ compared with patients without ionized hypomagnesemia.

Conclusions: There are age-related differences in normal ionized magnesium concentrations. Ionized hypomagnesemia is a common and clinically relevant occurrence among patients older than 1 month of age undergoing surgery for congenital heart disease. (J Thorac Cardiovasc Surg 2000;119:891-8)
$\mathrm{M}_{\mathrm{p}}$ agnesium $(\mathrm{Mg})$ is an important regulator of multiple cardiovascular processes, including myocardial conduction, myocardial contractility, transmembrane calcium flux, potassium transport, vascular smooth muscle tone, coronary vascular reactivity, and nitric oxide synthesis. ${ }^{1-4}$ Ninety-nine percent of $\mathrm{Mg}$ is

From the Departments of Cardiology ${ }^{\mathrm{a}}$ and Anesthesia, ${ }^{\mathrm{b}}$ Children's Hospital, and the Departments of Pediatrics ${ }^{\mathrm{c}}$ and Anaesthesia, ${ }^{\mathrm{d}}$ Harvard Medical School, Boston, Mass.

Supported in part by funds from the Nova Biomedical Corporation (Waltham, Mass) and the Boston Children's Heart Foundation.

Presented at the American College of Cardiology Forty-eighth Annual Scientific Session, New Orleans, La, March 7-10, 1999. distributed in the intracellular fluid, and $1 \%$ is distributed in the extracellular fluid. Circulating Mg exists in 3 forms: a protein-bound fraction ( $25 \%$ bound to albumin and $8 \%$ bound to globulins), a chelated fraction $(12 \%)$, and the physiologically active ionized fraction (IMg; 55\%). ${ }^{1}$ Total serum magnesium concentration

Received for publication March 22, 1999; revisions requested July 12, 1999; revisions received Oct 29, 1999; accepted for publication Oct 29, 1999.

Address for reprints: Ricardo Munoz, MD, Cardiac ICU Office, FA105, Children's Hospital, 300 Longwood Ave, Boston, MA 02115 (E-mail: munoz_r@a1.tch.harvard.edu).

Copyright $\odot 2000$ by The American Association for Thoracic Surgery 0022-5223/2000 $\$ 12.00+0 \quad \mathbf{1 2 / 1 / 1 0 4 3 3 7}$

doi: $10.1067 / \mathrm{mtc} .2000 .104337$ 
Table I. Composition of cardioplegic solutions

\begin{tabular}{lcc}
\hline & Plegisol solution & Custom-made solution \\
\hline $\mathrm{Mg}^{2+}$ & $32 \mathrm{mEq} / \mathrm{L}$ & $3 \mathrm{mEq} / \mathrm{L}$ \\
$\mathrm{Ca}^{2+}$ & $2.4 \mathrm{mEq} / \mathrm{L}$ & - \\
$\mathrm{K}^{+}$ & $16 \mathrm{mEq} / \mathrm{L}$ & $30 \mathrm{mEq} / \mathrm{L}$ \\
$\mathrm{Na}^{+}$ & $110 \mathrm{mEq} / \mathrm{L}$ & $140 \mathrm{mEq} / \mathrm{L}$ \\
$\mathrm{CL}^{-}$ & $160 \mathrm{mEq} / \mathrm{L}$ & $98 \mathrm{mEq} / \mathrm{L}$ \\
Gluconate & - & $23 \mathrm{mEq} / \mathrm{L}$ \\
\hline
\end{tabular}

(MgT), which includes the protein-bound, chelated, and ionized fractions, was the first and most easily measured form of $\mathrm{Mg}$ and is the clinical measurement routinely used for assessing circulating $\mathrm{Mg}$. Total serum hypomagnesemia has been associated with hypokalemia, hypocalcemia, cardiac arrhythmias, ischemic heart disease, myocardial injury, low cardiac output syndrome, hypertension, cardiomyopathy, sudden death, and muscular weakness. ${ }^{1,4,5}$ Total serum hypomagnesemia has been reported in up to $94 \%$ of adult patients during cardiopulmonary bypass (CPB), ${ }^{6}$ but the level is altered by factors such as $\mathrm{pH}$, serum protein concentrations, and illness severity. ${ }^{1}$ Furthermore, it has been recently demonstrated that patients with ionized hypomagnesemia may have normal levels of $\mathrm{MgT}^{3,7}$

Accurate measurement of IMg has only recently been available in clinical practice. Pediatric patients during CPB exhibit alterations in IMg. These alterations differ depending on both patient weight and the use of cardioplegic solution. ${ }^{8}$ In addition, preoperative medication to treat congestive heart failure and hemodilution on $\mathrm{CPB}$ may also contribute to ionized hypomagnesemia.

Because of previous technical limitations in the measurement of IMg and lack of normative data, there is little information regarding the frequency and clinical implications of ionized hypomagnesemia in patients undergoing $\mathrm{CPB}$ during operations for congenital cardiac disease. We hypothesized that ionized hypomagnesemia may be a common and clinically relevant problem in patients undergoing $\mathrm{CPB}$ during operations for congenital cardiac disease. To test this hypothesis, we measured IMg concentrations in 113 control patients to obtain reference values and in 186 patients undergoing $\mathrm{CPB}$ during operations for congenital heart disease.

\section{Patients and methods}

The protocol was approved by the Institutional Review Boards at Children's Hospital in Boston and Mount Auburn Hospital in Cambridge, Massachusetts. A total of 1303 blood samples were obtained from 299 subjects (113 control patients and 186 patients undergoing CPB during surgery for congenital cardiac disease). Patients were selected between April 1996 and April 1998 by using convenience sampling. We obtained written informed consent from parents or from the subjects older than 17 years of age.

Control patients. The control group included 15 healthy term neonates, 85 children undergoing noncardiac minor elective operative procedures, and 13 healthy adults. The 15 normal neonates had a venous blood sample drawn at 48 hours of life. Children had a venous blood sample drawn during the preoperative evaluation. Excluded from the control group were patients with any medical history of a disorder known to alter $\mathrm{Mg}$ metabolism, including malnutrition, alcoholism, nephropathies, asthma, sepsis, diabetes, pancreatitis, cardiac disease, endocrine disorder, malabsorption syndromes, and transfusion therapy. ${ }^{1}$ Also excluded from the control group were patients receiving medications known to interfere with $\mathrm{Mg}$ metabolism (eg, diuretics, digoxin, aminoglycosides, carbenicillin, cisplatinum, methotrexate, cyclosporine [INN: ciclosporin], ethanol, terbutaline, pentamidine, and citrate). ${ }^{1}$ None of the control subjects, including mothers of neonates, received $\mathrm{Mg}$ supplementation.

Surgical group. Study patients included 186 nonconsecutive subjects (77 female and 109 male subjects) undergoing operations for congenital cardiac disease. Depending on the surgeon's preference, patients received one of the following cardioplegic solutions: Plegisol (Abbott Laboratories, North Chicago, Ill) or a custom-made cardioplegic solution (Table I). For every four parts of custom-made cardioplegic solution, one part of pump blood was added. The composition of the bypass circuit and perfusion protocols were according to established institutional practices. The circuits were primed with Plasma-Lyte solution (Baxter, Deerfield, Ill) and whole blood if necessary to achieve a patient hematocrit level of greater than $20 \%$. All patients undergoing deep hypothermic CPB with either circulatory arrest or low flow were administered furosemide $0.25 \mathrm{mg} / \mathrm{kg}$ and methylprednisolone 30 $\mathrm{mg} / \mathrm{kg}$ as a single bolus within the first minute of CPB. All patients received mannitol $0.5 \mathrm{mg} / \mathrm{kg}$ at the time of aortic crossclamp removal.

After anesthesia was achieved, a baseline arterial blood sample was taken before CPB; additional arterial blood samples were drawn during both cooling and rewarming on CPB, immediately after separating from $\mathrm{CPB}$, and during admission to the cardiac intensive care unit (CICU). Arterial blood $(1 \mathrm{~mL})$ was collected into a heparinized blood gas syringe (25 USP of dry heparin per milliliter) and was immediately analyzed on the Ultra C analyzer (Nova Biomedical, Waltham, Mass) for IMg, blood gases, hematocrit level, electrolytes, $\mathrm{Ca}^{++}$, lactate, and glucose. Quality controls were performed on the analyzer before laboratory determinations.

Because of the range of diagnoses, ages, and surgical procedures, patients were compared at the time of admission to the CICU by using the pediatric severity of illness (PRISM III) score. ${ }^{9}$ This was calculated by recording blood pressure, heart rate, temperature, arterial blood gases, glucose, electrolytes, creatinine, blood urea nitrogen, white blood cells, 


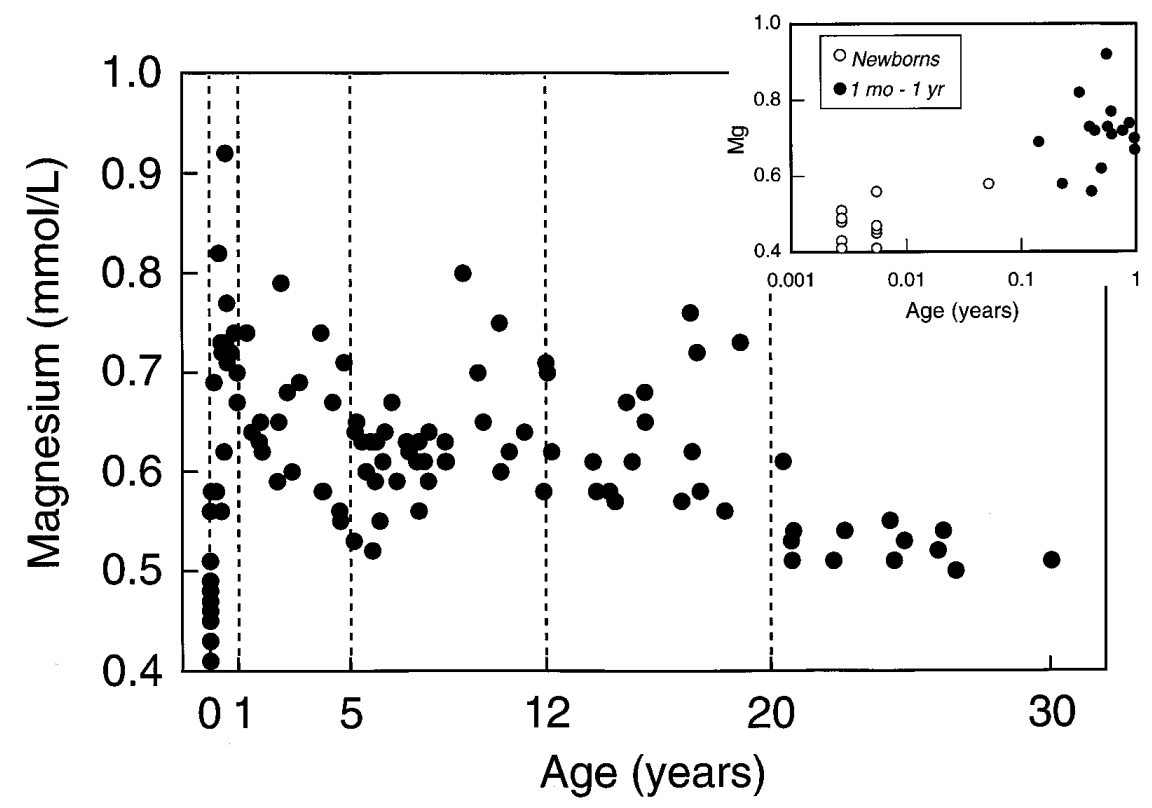

Fig 1. Scatter plot of IMg versus age for the control group. Vertical lines indicate age categories. Scatter of IMg levels between newborns and infants is shown in the inset graph.

prothrombin time, partial thromboplastin time, and platelets on CICU admission. Arrhythmias were recorded during anesthesia and after CICU admission by means of continuous electrocardiographic monitoring. In addition, medications, duration of mechanical ventilation, and CICU length of stay were recorded.

Ionized hypomagnesemia was defined as an IMg concentration more than 2 SDs below the mean IMg concentration of control subjects in the same age group. During CPB, ionized hypomagnesemia was defined as the average of all measurements during this period.

Statistical analysis. Because IMg concentrations were not normally distributed, a log transformation was used when defining ionized hypomagnesemia. Age-related differences were determined by using 1-way analysis of variance with a Bonferroni correction for multiple comparisons. Differences in proportions were analyzed by using the Fisher exact test. Medians were compared by using the Wilcoxon rank-sum test. Controlling for CPB time, the PRISM III score, duration of mechanical ventilation, and CICU stay were analyzed by means of multiple linear regression methods after performing a $\log$ transformation. Mortality was analyzed by logistic regression. All multivariable analyses were done for neonates and nonneonates separately. Analyses were performed by using the Stata statistical package (Stata Corporation, College Station, Tex).

\section{Results}

Control group. The IMg concentration varied with age and was significantly lower in neonates (Fig 1).
Means \pm SD for IMg are as follows: 1 month or less, $0.47 \pm 0.05 \mathrm{mmol} / \mathrm{L}$; greater than 1 month and less than 1 year, $0.71 \pm 0.09 \mathrm{mmol} / \mathrm{L} ; 1$ to 4 years, $0.65 \pm 0.07$ $\mathrm{mmol} / \mathrm{L} ; 5$ to 11 years, $0.62 \pm 0.05 \mathrm{mmol} / \mathrm{L} ; 12$ to 19 years, $0.64 \pm 0.06 \mathrm{mmol} / \mathrm{L}$; and 20 or more years, 0.53 $\pm 0.03 \mathrm{mmol} / \mathrm{L}$. Because there were no statistically significant differences among patients 1 to 19 years of age, these patients were analyzed as one group. Therefore 4 age groups provided our reference values from neonate to adulthood (Fig 2).

Surgical group. One hundred eighty-six patients were studied: 47 neonates (median age, 6 days; range 1-27 days); 48 infants (median age, 0.37 years; range, 0.09-0.96 years); 83 children (median age, 3.7 years; range, 1.1-19.3 years); and 8 adults 20 years of age or older (median age, 30 years; range, 20.7-39.8 years). The median age of all patients studied was 11.4 months (range, 1 day to 39.8 years). Types of surgical procedures are shown in Table II, and patient demographics and CPB details are shown in Table III. There were no statistically significant differences in frequency of ionized hypomagnesemia at baseline on the basis of sex $(P$ $=.9$ ). The overall mortality for the patients studied was 9\% (17/186). Because of patient selection bias (nonconsecutive patients), this mortality does not represent our actual institutional mortality for cardiac surgery over this period, which was $2.8 \%$.

Preoperative IMg. Compared with our control 


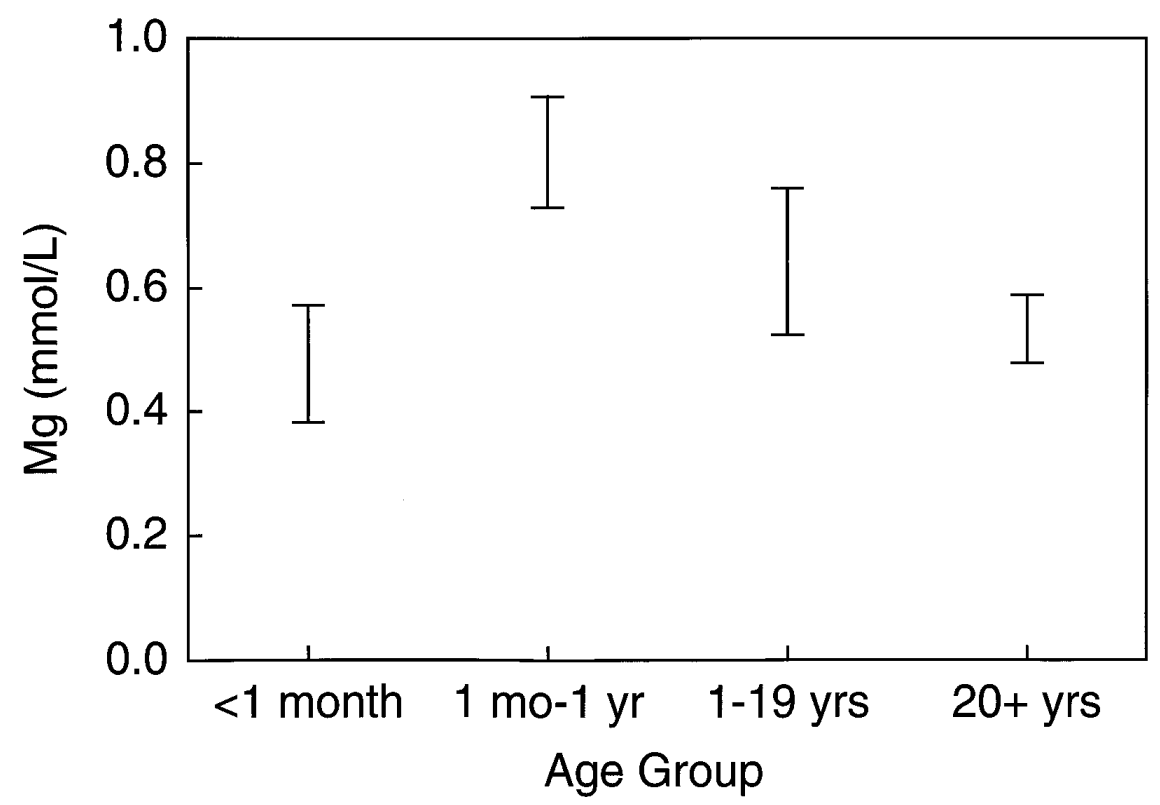

Fig 2. Reference values ( \pm 2 SDs of the log transformation) for whole blood ionized $\mathrm{Mg}$ concentrations by age group.

Table II. Surgical procedures

\begin{tabular}{lc}
\hline Surgical procedure & No. \\
\hline ASD/VSD & 53 \\
Right ventricular outflow tract repair & 26 \\
Valvuloplasty & 10 \\
Arterial switch operation & 13 \\
Norwood operation & 16 \\
Bidirectional Glenn anastomosis & 13 \\
Modified Fontan procedure & 13 \\
Atrioventricular canal repair & 8 \\
Left ventricular outflow tract repair & 4 \\
Cardiac transplantation & 4 \\
Coarctation of the aorta and VSD & 4 \\
Total anomalous pulmonary venous connection & 2 \\
Others & 20 \\
Total & 186 \\
\hline
\end{tabular}

$A S D$, Atrial septal defect; $V S D$, ventricular septal defect.

group, preoperative ionized hypomagnesemia was a common finding in our patients with congenital cardiac defects who were older than 1 month of age, occurring in $41 \%$ of patients. The percentage of neonates with preoperative ionized hypomagnesemia was significantly lower than that of older patients (10\% vs $41 \%, P<.001$; Fig 3 ).

Preoperative medications were being taken by 79 $(42 \%)$ of 186 patients, most commonly digoxin $(n=47)$, furosemide $(n=57)$, or captopril $(n=27)$. In neonates there was no association between preoperative use of furosemide and frequency of ionized hypomagnesemia during CPB. In contrast, a greater proportion of patients older than 1 month taking furosemide before surgery had ionized hypomagnesemia during CPB than patients taking other or no medications ( $55 \%$ vs $34 \%, P=.04$ ).

Intraoperative IMg. The changes in IMg levels during the measured time points are shown in Table IV. There was a decrease in IMg levels after the onset of CPB in neonates and infants. However, by the end of bypass, IMg had returned to the preoperative level.

Patients who received the custom-made cardioplegic solution exhibited a higher incidence of ionized hypomagnesemia during (48\% vs $27 \%, P=.01)$ and after CPB (39\% vs $17 \%, P=.003)$ compared with patients who received Plegisol solution. Nevertheless, there were no statistically significant differences in hemodynamic variables, inotropic support, PRISM III score, duration of mechanical ventilation, CICU stay, and mortality between patients who received custom-made cardioplegic solution and Plegisol solution.

CICU outcome. The differences in PRISM III score, duration of mechanical ventilation and CICU stay, and incidence of postoperative arrhythmias in patients with and without ionized hypomagnesemia are shown in Table V. Thirty-three (18\%) of 186 patients had arrhythmias (ventricular, $\mathrm{n}=9$; atrial, $\mathrm{n}=24$ ) during the postoperative period. The incidence of arrhythmia was not associated with ionized hypomagnesemia during CPB (Table V). 


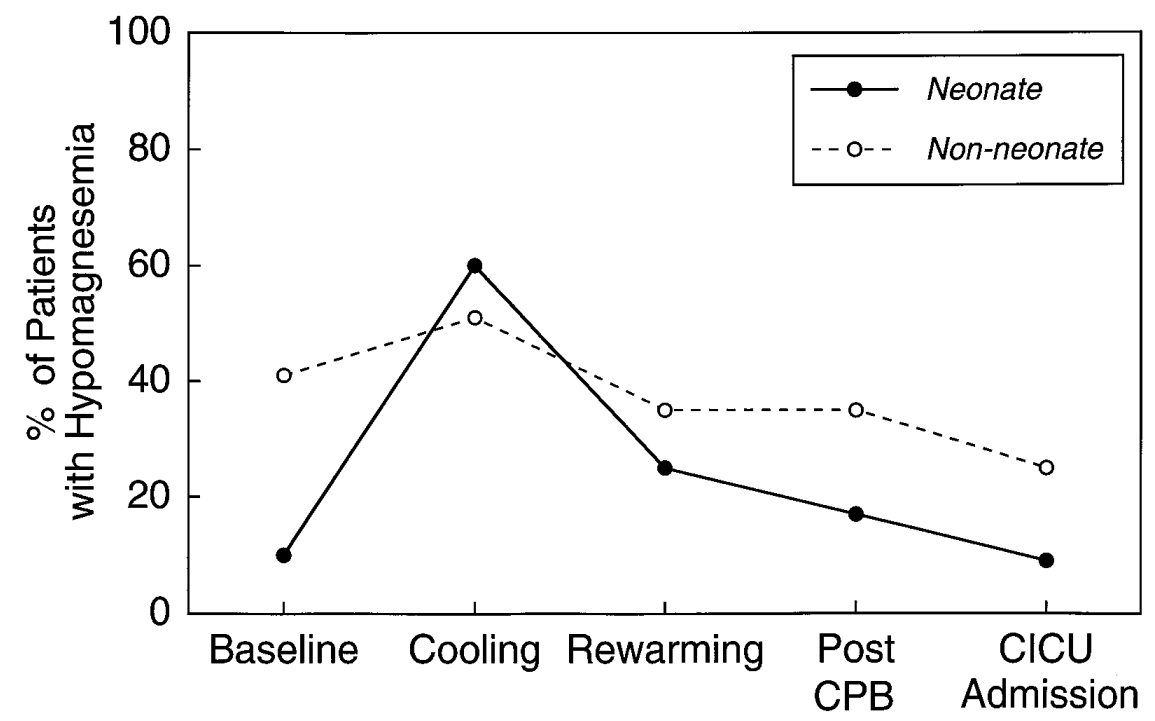

Fig 3. The prevalence of hypomagnesemia in neonates and patients older than 1 month during the perioperative period.

Table III. Age, weight, sex, and CPB characteristics by age group

\begin{tabular}{lccccc}
\hline & \multicolumn{1}{c}{ Total } & $\leq 1$ mo & 1 mo-1 y & $1-19 y$ & $\geq 20 y$ \\
\hline Number & 186 & 47 & 48 & 83 & 8 \\
Age & $11.4 \mathrm{mo}(27 \mathrm{~d}-3.8 \mathrm{y})$ & $6 \mathrm{~d}(4-10 \mathrm{~d})$ & $4.4 \mathrm{mo}(2.3-7.7 \mathrm{mo})$ & $3.7 \mathrm{y}(2.3-9.9 \mathrm{y})$ & $30.3 \mathrm{y}(22.5-37.5 \mathrm{y})$ \\
Weight (kg) & $7.3(3.8-16)$ & $3.2(2.7-3.6)$ & $5.4(4.4-6.7)$ & $14(11-32.7)$ & $62.5(53-66.9)$ \\
Sex (M/F) & $109 / 77$ & $25 / 2$ & $28 / 20$ & $51 / 32$ & $5 / 3$ \\
Prior medical therapy, n (\%) & $79(42.5)$ & $20(42.5)$ & $28(58.3)$ & $27(32.5)$ & $4(50)$ \\
CPB duration (min) & $101(68-139)$ & $123(105-147)$ & $98(73-145)$ & $80(55-120)$ & $102(72-160)$ \\
Crossclamp time (min) & $52(33-73)$ & $70(46-79)$ & $52(34-80)$ & $44(25-63)$ & $63(50-107)$ \\
Circulatory arrest & & & & $3(3.6 \%)$ & $1(12.5)$ \\
$\quad$ Patients, n (\%) & $65(34.9)$ & $41(87.2)$ & $20(41.7)$ & $29(16-42)$ & $32(32-32)$ \\
Duration (min) & $29(14-43)$ & $30(16-49)$ & $24(9-38)$ & & \\
\hline
\end{tabular}

Data are expressed as median (25th-75th percentile) where applicable. $C P B$, Cardiopulmonary bypass.

Table IV. IMg levels across the perioperative period by age group

\begin{tabular}{llllll}
\hline & \multicolumn{1}{c}{ Total } & \multicolumn{1}{c}{$\leq 1 \mathrm{mo}$} & \multicolumn{1}{c}{$1 \mathrm{mo-1} y$} & \multicolumn{1}{c}{$1-19 y$} & $\geq 20 y$ \\
\hline Reference range (mmol/L) & $0.61(0.55-0.67)$ & $0.46(0.43-0.49)$ & $0.72(0.67-0.74)$ & $0.63(0.59-0.67)$ & $0.53(0.51-0.54)$ \\
Preoperative & $0.54(0.49-0.58)$ & $0.48(0.42-0.57)$ & $0.56(0.53-0.6)$ & $0.54(0.51-0.57)$ & $0.54(0.51-0.58)$ \\
Cooling CPB & $0.43(0.27-0.59)$ & $0.33(0.22-0.43)$ & $0.30(0.25-0.44)$ & $0.58(0.43-0.66)$ & $0.58(0.55-0.78)$ \\
Rewarming CPB & $0.56(0.46-0.74)$ & $0.47(0.38-0.53)$ & $0.49(0.43-0.64)$ & $0.70(0.53-1.2)$ & $0.65(0.55-0.93)$ \\
Post-CPB & $0.56(0.47-0.71)$ & $0.48(0.4-0.56)$ & $0.51(0.44-0.63)$ & $0.65(0.53-0.93)$ & $0.56(0.53-0.97)$ \\
CICU admission & $0.60(0.52-0.68)$ & $0.58(0.45-0.67)$ & $0.59(0.53-0.66)$ & $0.62(0.54-0.71)$ & $0.59(0.56-0.71)$ \\
\hline
\end{tabular}

Data are expressed as median (25th-75th percentile) as applicable. $C P B$, Cardiopulmonary bypass; $C I C U$, cardiac intensive care unit.

Because neonates had longer median CPB time compared with patients older than 1 month (123 vs 87 minutes, $P<.0001)$, they were analyzed separately. In the multivariable analysis controlling for CPB time among patients older than 1 month, the duration of mechanical ventilation and CICU stay were longer in those who were hypomagnesemic at baseline, during $\mathrm{CPB}$, and after CPB (Table VI). There was a tendency to have a higher PRISM III score in patients who were hypomagnesemic at baseline and during CPB. In contrast to patients older than 1 month, neonates with hypomagnesemia did not have a significantly higher PRISM III 
Table V. Difference in age, PRISM III score, duration of mechanical ventilation, CICU stay, arrhythmias, hypokalemia, and hypocalcemia in patients with and without hypomagnesemia during $C P B^{*}$

\begin{tabular}{lccc}
\hline \multicolumn{1}{c}{ Yes } & No & P value \\
\hline No. & 73 & 108 & - \\
Age (y) & $0.4(33 \mathrm{~d}-1 \mathrm{y})$ & $2.6(20 \mathrm{~d}-9.2 \mathrm{y})$ & .0002 \\
PRISM III score & $6(2-8)$ & $5(2-7.5)$ & .05 \\
Duration of mechanical ventilation (h) & $31.4(16-71)$ & $19(9-50)$ & .02 \\
CICU stay (d) & $2.9(2-5)$ & $2(1-4)$ & .02 \\
Postoperative arrhythmia & $9(12.3)$ & $23(21.3)$ & .16 \\
$\quad$ Total, $\mathrm{n}(\%)$ & $3(4.1)$ & $5(4.6)$ & 1 \\
Ventricular, n (\%) & $6(8.2)$ & $18(16.7)$ & .12 \\
Atrial, $\mathrm{n}(\%)$ & $58(79)$ & $64(59)$ & .01 \\
Hypo-Ca & $6(6)$ & .04 \\
Hypo- $\mathrm{K}^{+}$during CPB, $\mathrm{n}(\%)$ & $11(15)$ & $11(10)$ & .8 \\
Mortality, n (\%) & $6(8)$ & & \\
\hline
\end{tabular}

Data are expressed as median (25th-75th percentile) as applicable. Yes, Patients who were hypomagnesemic during CPB irrespective of age group; No, patients who were not hypomagnesemic during $\mathrm{CPB}$ irrespective of age group; $C I C U$, cardiac intensive care unit; $C P B$, cardiopulmonary bypass.

*Five patients did not have IMg measured during CPB.

Table VI. Relationships of outcome with ionized hypomagnesemia among patients older than 1 month, controlling for CPB time*

\begin{tabular}{|c|c|c|c|c|c|c|c|c|}
\hline & \multicolumn{2}{|c|}{ Before $C P B$} & \multicolumn{2}{|c|}{ During $C P B$} & \multicolumn{2}{|c|}{ After $C P B$} & \multicolumn{2}{|c|}{ CICU admission } \\
\hline & $\begin{array}{c}\text { Regression } \\
\text { coefficient } \pm S E\end{array}$ & $\mathrm{P}$ value & $\begin{array}{c}\text { Regression } \\
\text { coefficient } \pm S E\end{array}$ & $\mathrm{P}$ value & $\begin{array}{c}\text { Regression } \\
\text { coefficient } \pm S E\end{array}$ & $\mathrm{P}$ value & $\begin{array}{c}\text { Regression } \\
\text { coefficient } \pm S E\end{array}$ & $\mathrm{P}$ value \\
\hline \multicolumn{9}{|c|}{$\begin{array}{l}\text { Duration of mechanical } \\
\text { ventilation }(\mathrm{h})\end{array}$} \\
\hline Hypo-IMg & $0.56 \pm 0.24$ & .02 & $0.81 \pm 0.23$ & .001 & $0.56 \pm 0.25$ & .03 & $0.45 \pm 0.28$ & .11 \\
\hline CBP time & $0.011 \pm 0.002$ & $<.001$ & $0.011 \pm 0.002$ & $<.001$ & $0.010 \pm 0.002$ & $<.001$ & $0.011 \pm 0.002$ & $<.001$ \\
\hline \multicolumn{9}{|l|}{ CICU stay (d) } \\
\hline Нypo-IMg & $0.41 \pm 0.14$ & .003 & $0.36 \pm 0.14$ & .01 & $0.29 \pm 0.15$ & .05 & $0.18 \pm 0.16$ & .3 \\
\hline CBP time & $0.0067 \pm .0012$ & $<.001$ & $0.0068 \pm 0.0012$ & $<.001$ & $0.0061 \pm .0013$ & $<.001$ & $0.0069 \pm 0.0012$ & $<.001$ \\
\hline \multicolumn{9}{|c|}{ PRISM III score } \\
\hline Hypo-IMg & $0.16 \pm 0.12$ & .16 & $0.17 \pm 0.11$ & .14 & $0.22 \pm 0.12$ & .07 & $0.3 \pm 0.13$ & .02 \\
\hline CPB time & $0.0047 \pm 0.001$ & $<.001$ & $0.0047 \pm 0.001$ & $<.001$ & $0.0043 \pm 0.0011$ & $<.001$ & $0.0046 \pm 0.001$ & $<.001$ \\
\hline
\end{tabular}

$C I C U$, Cardiac intensive care unit; $C P B$, cardiopulmonary bypass; Hypo-IMg, ionized hypomagnesemia.

*The duration of mechanical ventilation, CICU stay, and PRISM III score were analyzed after performing a log transformation.

score, increased duration of mechanical ventilation, or prolonged CICU stay.

Ionized hypomagnesemia was associated with hypokalemia $(P=.04)$ and hypocalcemia $(P=.01)$ during CPB (Table V). After controlling for hypokalemia and hypocalcemia, the relationship between hypomagnesemia and duration of mechanical ventilation and CICU stay did not change.

\section{Discussion}

Control group. In contrast to previous reports, ${ }^{10,11}$ our study demonstrates age-related differences in whole blood IMg concentrations. Newborns exhibited the lowest IMg concentration, and infants exhibited the highest IMg concentration within the whole group. The
IMg concentrations we measured in neonates are similar to values recently reported in healthy neonates at birth and 24 hours of life, ${ }^{12}$ although the IMg values in our study were measured at 48 hours of life. Our reference values for IMg may therefore not represent the entire neonatal period, and further studies are necessary to define the range of normal values in this age group. This may explain the lack of association between ionized hypomagnesemia and the measured outcome variables in our surgical neonate group.

The causes of age-related differences in IMg concentrations were not assessed in our study. Renal losses and changes in body fluid compartment and diet may play a role in determining these differences. The ionized fraction represents the physiologically active por- 
tion of the cation, and MgT is neither sensitive nor specific in predicting the ultrafilterable (ie, ionized plus chelated) fraction of Mg. ${ }^{1}$ Age, serum albumin concentration, $\mathrm{pH}$, and serum salts may alter the distribution of circulating $\mathrm{Mg}$ among the ionized, chelated, and protein fraction. Although we did not measure serum protein concentration, previous studies have shown no significant differences in serum protein concentrations among different age groups. ${ }^{13}$

Surgical group. The results of this study indicate that ionized hypomagnesemia is a common and clinically relevant problem in patients undergoing CPB during operations for congenital cardiac disease. Ionized hypomagnesemia may be present before the operation and can also occur after hemodilution on CPB and administration of cardioplegic solution.

Before surgery, $34 \%$ of our patients had ionized hypomagnesemia. The cause of ionized hypomagnesemia in this group of patients may be multifactorial. Forty-two percent of our patients had taken at least one cardiac medication (diuretics, vasodilators, or digoxin). Digoxin impairs the $\mathrm{Mg}$-dependent, cell membrane, sodium-potassium, adenosine-triphosphatase pump, inhibiting transcellular $\mathrm{Mg}$ transport and perhaps renal tubular $\mathrm{Mg}$ reabsorption. ${ }^{14}$ Similarly, diuretics may increase renal loss of $\mathrm{Mg}$ leading to hypomagnesemia. ${ }^{14}$ In our study intraoperative ionized hypomagnesemia was more prevalent in those patients taking furosemide before surgery.

Another factor that could contribute to ionized hypomagnesemia is suboptimal nutritional support and failure to thrive before surgery. Reduced oral feeding and the administration of intravenous fluids supplying inadequate $\mathrm{Mg}$ concentrations might be important factors favoring the development of ionized hypomagnesemia in preoperative patients.

It has been reported that alterations in whole blood IMg concentration occur during CPB in adults. ${ }^{8}$ However, because of lack of normative data in children, the true prevalence and clinical relevance of ionized hypomagnesemia during CPB has been unknown, although our study indicates that ionized hypomagnesemia is common during CPB.

The CPB circuit prime volume in neonates and infants is large compared with the patient's circulating blood volume. ${ }^{15}$ In our study neonates exhibited the highest frequency of ionized hypomagnesemia during cooling, which could reflect hemodilution. Chelation of IMg by acid-citrate-dextrose when donated blood is used to prime the $\mathrm{CPB}$ circuit and could also contribute to a fall in IMg. ${ }^{16}$

Although we did not demonstrate a statistically sig- nificant difference in postoperative outcome between the two cardioplegic solutions, ionized hypomagnesemia was more common in patients who received the custom-made cardioplegic solution than in patients who received Plegisol solution. After CPB, normalization of myocardial cell function is dependent on the restoration of oxidative metabolism (repletion of cellular oxidative adenosine triphosphate stores). $\mathrm{Mg}$ is an absolute requirement of oxidative phosphorylation, and $\mathrm{Mg}$ deficiency may contribute to depletion of adenosine triphosphate and subsequent poor myocardial performance. ${ }^{17}$ Improved left ventricular systolic volume index after intravenous $\mathrm{Mg}$ administration has been demonstrated in adults after coronary artery bypass grafting. $\mathrm{Mg}$ supplementation in pediatric patients undergoing surgery for congenital cardiac disease during the perioperative period could also enhance myocardial recovery, but specific prospective studies are necessary.

Contrary to other reports, ${ }^{1,6}$ ionized hypomagnesemia during CPB was not associated with an increase in frequency of ventricular arrhythmias in our patients (8/181). Although supraventricular arrhythmias were more common (24/181), once again the IMg level did not appear to be a contributory factor.

Hypomagnesemia is often associated with other electrolyte abnormalities, such as hypocalcemia and hypokalemia, ${ }^{1,17-19}$ and in our study, patients with ionized hypomagnesemia were more likely to have hypocalcemia and hypokalemia during CPB and after CPB than were those without ionized hypomagnesemia. The dysfunction of cell membrane sodium-potassiumadenosine-triphosphatase caused by ionized hypomagnesemia may allow efflux of potassium ions from the cell, leading to intracellular hypokalemia. ${ }^{1}$ Moreover, the kidney may be unable to adequately reabsorb potassium during hypomagnesemic states, increasing the renal loss of potassium. ${ }^{20}$ Diuretic therapy used in the preoperative period of patients undergoing cardiac surgery may be responsible for concomitant urinary $\mathrm{Mg}$ and potassium losses. ${ }^{1,14}$ Despite the relationship between ionized hypomagnesemia, hypocalcemia, and hypokalemia, neither hypocalcemia nor hypokalemia changed the effect of ionized hypomagnesemia on CICU stay, duration of mechanical ventilation time, or PRISM III score at CICU admission.

Although the duration of CPB may influence the outcomes measured in our study, after controlling for the duration of CPB by using linear regression methods, patients in our study older than 1 month of age who were hypomagnesemic during CPB had a longer period of mechanical ventilation, longer CICU stay, and a 
tendency for a higher PRISM III score on CICU admission.

Skeletal and respiratory muscle weakness has been associated with hypo-MgT in critically ill neonates and adults, ${ }^{1,21}$ and it is possible that respiratory muscle weakness might be in part a contributing factor to the increased duration of mechanical ventilation.

\section{Limitation of the study}

Because of the nonrandom method of patient selection, there was a selection bias toward patients with more complicated congenital abnormalities with higher mortality (study mortality of $9 \%$ of 186 patients), which does not reflect our true institutional mortality over that period (2.8\% for 1332 patients undergoing CPB). Selection of high-risk patients may have increased the frequency of hypomagnesemia and its correlation with adverse outcome.

\section{Conclusion}

Our findings indicate that there are age-related differences in normal IMg concentration. Therefore ionized hypomagnesemia should be defined on the basis of normative data for neonates, infants, children, and adults. Perioperative ionized hypomagnesemia is a common and possibly clinically relevant occurrence among patients undergoing repair of congenital heart defects. Patients older than 1 month who had ionized hypomagnesemia during CPB had a higher PRISM III score at CICU admission, a longer period of mechanical ventilation, and a longer CICU stay than did patients without ionized hypomagnesemia. Our observations merit a randomized trial of perioperative $\mathrm{Mg}$ administration.

\section{REFERENCES}

1. Salem M, Munoz R, Chernow B. Hypomagnesemia in critical illness: a common and clinically important problem. Crit Care Clin 1991;7:225-2.

2. Pearson PJ, Evora PRB, Seccombe JF, Schaff HV. Hypomagnesemia inhibits nitric oxide release from coronary endothelium: protective role of magnesium infusion after cardiac operations. Ann Thorac Surg 1998;65:967-72.

3. Maggioni A, Orzalesi M, Mimouni FB. Intravenous correction of neonatal hypomagnesemia: effect on ionized magnesium. J Pediatr 1998;132:652-5.
4. Storm W, Zimmerman JJ. Magnesium deficiency and cardiogenic shock after cardiopulmonary bypass. Ann Thorac Surg 1997;64: 572-7.

5. Caspi J, Rudis E, Bar I, Safadi T, Saute M. Effects of magnesium on myocardial function after coronary artery bypass grafting. Ann Thorac Surg 1995;59:942-7.

6. England MR, Gordon G, Salem M, Chernow B. Magnesium administration and dysrrhythmias after cardiac surgery. JAMA 1992;268:2395-402.

7. Fiser RT, Torres A Jr, Butch AW, Valentine JL. Ionized magnesium concentrations in critically ill children. Crit Care Med 1998; 26:2048-52.

8. Fox ML, Burrows FA, Reid RW, Hickey PR, Laussen PC, Hansen DD. The influence of cardiopulmonary bypass on ionized magnesium in neonates, infants, and children undergoing repair of congenital heart lesions. Anesth Analg 1997;84:497-500.

9. Pollack MM, Patel KM, Ruttimann UE. PRISM III: an updated pediatric risk of mortality score. Crit Care Med 1996;24:743-52.

10. Mays JE Jr, Keele DK. Serum magnesium levels in healthy children and various disease states. Am J Dis Child 1961;102:623-54.

11. Anast CS. Serum magnesium levels in the newborn. Pediatrics 1964;33:969-74.

12. Cook LA, Mimouni FB. Whole blood ionized magnesium in the healthy neonate. J Am Coll Nutr 1997;16:181-3.

13. Rothschild MA, Oratz M, Schreiber SS. Serum albumin. Hepatology 1988;8:385-401.

14. Quamme GA, Dirks JH. Renal magnesium transport. Rev Physiol Biochem Pharmacol 1983;97:69-110.

15. Kern FH, Morana NJ, Sears JJ, Hickey PR. Coagulation defects in neonates during cardiopulmonary bypass. Ann Thorac Surg 1992;54:541-6.

16. Aglio LS, Stanford GG, Maddi R, Boyd III JL, Nussbaum S, Chernow B. Hypomagnesemia is common following cardiac surgery. J Cardiothorac Vasc Anesth 1991;5:201-8.

17. Leier CV, Dei Cas L, Metra M. Clinical relevance and management of the major electrolyte abnormalities in congestive heart failure: hyponatremia, hypokalemia, and hypomagnesemia. Am Heart J 1994;128:564-74.

18. Aziz S, Haigh WG, Van Norman GA, Kenny RJ, Kenny MA. Blood ionized magnesium concentrations during cardiopulmonary bypass and their correlation with other circulating cations. J Card Surg 1996;11:341-7.

19. Garty A, Alkalay A, Bernheim JL. Parathyroid hormone secretion and responsiveness to parathyroid hormone in primary hypomagnesemia. Isr J Med Sci 1983;19:345-8.

20. Ryzen E. Magnesium homeostasis in critically ill patients. Magnesium 1989;8:201-12.

21. Fiaccadori E, Del Canale S, Coffrini E, Melej R, Vitali P, Guariglia A, et al. Muscle and serum magnesium in pulmonary intensive care unit patients. Crit Care Med 1988;16:751-60. 
The Journal of Thoracic and

Munoz et al 899

Cardiovascular Surgery

Volume 119, Number 5 